\title{
Estados, espacios y hegemonía en la reestructuración global capitalista. Contribuciones para una perspectiva estructural, histórica y dinámica
}

\author{
JOEL SIDLER* \\ VÍCTOR RAMIRO FERNÁNDEZ**
}

Artículo recibido: 21 de junio de 2021

Artículo aprobado: 29 de noviembre de 2021

Doi: https://doi.org/10.12804/revistas.urosario.edu.co/desafios/a.10701

Para citar este artículo: Sidler, J., \& Fernández, V. R. (2022). Estados, espacios y hegemonía en la reestructuración global capitalista. Contribuciones para una perspectiva estructural, histórica y dinámica. Desafíos, 34(1), 1-35. https://doi.org/10.12804/revistas.urosario.edu. co/desafios/a.10701

\section{Resumen}

Con perspectivas no siempre convergentes, diversos corpus teóricos se han propuesto comprender las transformaciones que se observan desde hace cinco décadas en las dinámicas entre los Estados, los espacios de ejercicio del poder politico y el despliegue y la crisis de la hegemonía a nivel global. Este articulo presenta dos perspectivas

\footnotetext{
* Universidad Nacional del Litoral. IHUCSO Litoral (UNL-CONICET). Correo electrónico: sidlerjw@gmail.com. ORCID: https://orcid.org/0000-0001-8285-5499

** Universidad Nacional del Litoral. IHUCSO Litoral (UNL-CONICET). Correo electrónico: rfernand@ffjs.unl.edu.ar. ORCID: https://orcid.org/0000-0002-8650-8934
} 


\title{
2 I Joel Sidler - Víctor Ramiro Fernández
}

que intentan dar cuenta de estos elementos. Por un lado, la perspectiva sistémicoestructural bistórica, que reúne los aportes de la economía política internacional crítica y del análisis del sistema mundo; por el otro, la perspectiva posestructural, relacional que incluye estudios del campo de la geografía política crítica. Luego de exponer las tensiones y complementos entre estas se sostiene la necesidad y posibilidad de construir una perspectiva estructural, bistórica y dinámica, que busca enmarcar los desafíos y oportunidades para las estatidades en la región latinoamericana frente a la reestructuración global capitalista.

Palabras clave: capitalismo; teoría política; hegemonía; periferia; América Latina.

\section{States, Spaces, and Hegemony in the Global Capitalist Restructuring. Some Contributions towards a Structural, Historical, and Dynamic Perspective}

\begin{abstract}
Various theoretical approaches have attempted to understand the transformations that have been taking place over the last five decades in terms of the dynamics between states, how political power is exercised and the deployment and crisis of global hegemony. Such approaches have come from different perspectives that do not always converge. This paper discusses two approaches attempting to account for these elements. On the one hand, a historical structural systemic perspective, which brings together the contributions of the critical International Political Economy and World-Systems analysis. On the other, a relational poststructural perspective that includes studies from the field of critical political geography. After exposing the tensions and complements between them, we argue in favor of the possibility and need of constructing a structural, historical and dynamic perspective that seeks to frame the challenges and opportunities for statehood in Latin America in the face of global capitalist restructuring.
\end{abstract}

Keywords: Capitalism; political theory; hegemony; periphery; Latin America. 


\title{
Estados, espaços e hegemonia na reestruturação capitalista global. Contribuições para uma perspectiva estrutural histórica e dinâmica
}

\begin{abstract}
Resumo
Com perspectivas nem sempre convergentes, diversos corpus teóricos têm se proposto a compreender as transformações que vêm sendo observadas há cinco décadas na dinâmica entre os Estados, os espaços de exercício do poder político, o desdobramento e a crise de hegemonia em nivel global. Este artigo apresenta duas perspectivas que procuram dar conta desses elementos, de um lado, a perspectiva sistêmica estrutural histórica, que reúne as contribuições da crítica Economia Politica Internacional e da análise do Sistema Mundo; de outro, a perspectiva pós-estrutural relacional que inclui estudos do campo da geografia politica crítica. Depois de expor as tensões e complementos entre elas, sustenta-se a necessidade e a possibilidade de se construir uma perspectiva estrutural, histórica e dinâmica, que busca enquadrar os desafios $e$ as oportunidades da constituição do Estado na região latino-americana diante da reestruturação capitalista global.
\end{abstract}

Palavras-chave: capitalismo; teoria politica; hegemonia; periferia; América Latina.

\section{Introducción}

Las transformaciones en el sistema capitalista, la crisis de la hegemonía estadounidense y los múltiples cambios espaciales y funcionales en los Estados llaman a reconstruir y reactualizar las herramientas analíticas con el fin de comprender los márgenes de acción y las posibilidades y el rol de los Estados-nación, en general, y de la periferia, en particular. Partiendo de reconocer que, con enfoques no siempre convergentes, diversos corpus teóricos han intentado dar cuenta de estos cambios, en este artículo se presentan afinidades y tensiones entre dos formas de abordarlo: la perspectiva sistémico-estructural histórica, por un lado, y la posestructural relacional por el otro. De las interrelaciones entre ambas se conforma la perspectiva estructural, histórica y dinámica. 


\section{4 / Joel Sidler - Víctor Ramiro Fernández}

En particular, se analizó en términos conceptuales uno de los posibles hilos conductores al interior de cada perspectiva: la manera en que se conforma y redefine la hegemonía en su vínculo con el Estado y el espacio. Desde esta óptica, los tres conceptos (hegemonía, Estados y espacios) encuentran una relación que se retroalimenta y habilita el diálogo entre las perspectivas. Ello refiere a que las distintas maneras de comprender los vínculos entre Estado y espacio cambian nuestro entendimiento sobre su constitución y la forma de observarla, así como también sobre el concepto de hegemonía: cómo se construye y cómo se ejerce. Al poner en movimiento estas interrelaciones surge una renovada arquitectura conceptual que se beneficia de los insumos de ambas perspectivas al tiempo que incorpora otras al posicionarse desde una mirada periférica.

El artículo se divide en tres partes. En la primera y segunda se presentan las mencionadas perspectivas, con énfasis en sus análisis en torno a los tres conceptos de nuestro interés. Dentro de la primera perspectiva ubicamos dos vertientes: los aportes de la economía política internacional en su variante crítica, con los escritos de Robert Cox $(1981,1987)$, Stephen Gill $(1995,2008)$ y de este último junto a Douglas Law (1989), y recuperamos el análisis del sistema mundo desde las contribuciones de Immanuel Wallerstein $(1974,2004)$ y Giovanni Arrighi (1999, 2005, 2007). La segunda perspectiva incluye aportes de la geografía y la sociología política, junto con una revitalizada noción de espacialidad del ejercicio del poder político a nivel global. En ella se destacan las investigaciones de John Agnew (1994, 2005), Saskia Sassen (2008, 2013), Manuel Castells (2016) y John Allen (2003). Ambas perspectivas, con sus particularidades, aportan significativamente a la comprensión de los procesos globales que involucran el ejercicio de una hegemonía global, su interrelación con el Estado y las formas que adopta en el despliegue espacial.

La tercera parte alberga el posicionamiento con respecto a ellas y la selección de elementos necesarios para la perspectiva estructural, histórica y dinámica. Allí se analizan las potencialidades de recuperar ambas perspectivas desde sus tensiones y complementariedades para pensar cómo, en la actualidad y desde la periferia, los Estados 
se redefinen y rearticulan a partir del funcionamiento sistémico de las lógicas del capital a nivel global, y en qué medida nuevos actores y nuevas lógicas espaciales y temporales de funcionamiento del capitalismo afectan y reelaboran los modos de configuración de lo hegemónico. Se busca, por lo tanto, situar al Estado, el espacio y la hegemonía entre los flujos que los interpelan y redefinen y las estructuras que los contienen. Por último, se presentan breves conclusiones.

\section{Parte 1: Perspectiva sistémico-estructural histórica}

El origen compartido por las dos vertientes al interior de esta perspectiva impone una breve referencia. Tanto el análisis del sistema mundo (Arrighi, 2005) como la economía política internacional, en su variante crítica (Cox, 1983), construyen conceptualmente sobre los aportes de Antonio Gramsci, quien ha dejado su huella como militante y uno de los teóricos políticos más relevantes del siglo Xx. Con sus Cuadernos de la cárcel, escritos entre 1929 y 1935, propuso reflexiones que aún hoy permanecen como insumos centrales de diversas disciplinas en ciencias sociales.

La posición gramsciana, en un doble rechazo tanto de la posición fenomenista como instrumental (Buci-Glucksmann, 1978), esboza una teoría del Estado que no lo asume como reflejo ni instrumento, sino junto con los aparatos de hegemonía como condición de existencia y de funcionamiento de la estructura. Como señala Thwaites Rey (2007), la ampliación del concepto de Estado inicia en Gramsci con la incorporación de la hegemonía y su aparato. El marxista sardo rechazó las definiciones reduccionistas señalando que "por Estado debe entenderse además del aparato gubernamental también el aparato 'privado' de hegemonía o sociedad civil" (Gramsci, 1981a, p. 105). Este concepto ampliado de Estado le permitió a Gramsci (1981b), en el cuaderno 29, comprender cómo las clases dirigentes se unifican en este y ejercen su supremacía de dos maneras: «como 'dominio' y como 'dirección intelectual y moral’» (p. 387). Este autor también señala que "en la noción general de Estado entran elementos que deben reconducirse a la noción de 


\section{6 / Joel Sidler - Víctor Ramiro Fernández}

sociedad civil (en el sentido, podría decirse: Estado = sociedad política + sociedad civil, o sea hegemonía acorazada de coerción)" (Gramsci, 1981a, p. 75).

En consecuencia, el concepto gramsciano de Estado se comprende como una cristalización de una relación de fuerzas, a partir de la cual las clases dominantes no solo ejercen su dominio, sino que también logran obtener el consenso y compromiso de las clases dominadas para participar en la producción y reproducción del sistema. Así, entran en escena dos elementos centrales para la concepción gramsciana de hegemonía: el consenso y la coerción. Esta hegemonía no debe ser igualada con una ideología dominante, por ello no radica únicamente en lo que Althusser (1970) ha denominado los aparatos ideológicos del Estado. Por el contrario, la hegemonía y su aparato, para ser tales, deben funcionar a nivel económico, político y cultural. Es decir, tanto al nivel de las relaciones de producción como de reproducción. Cuando una determinada relación de fuerzas se establece en equilibrio, que en sentido gramsciano es un equilibrio siempre contradictorio, es cuando surge un bloque histórico. El Estado tiene un rol central en su constitución, que se da no porque las condiciones objetivas estén presentes, sino porque estas se construyen y se mantienen.

Este conjunto de conceptos será vital para la primera perspectiva, ya que tanto el giro gramsciano de la economía política internacional como el análisis del sistema mundo realizan una recuperación conceptual que sienta las bases para una comprensión general del funcionamiento de los aparatos de hegemonía dentro de la dinámica histórico-sistémica. Tal perspectiva es sistémico-estructural porque habilita una investigación enfocada en el movimiento general, con una mirada holística de los procesos de ordenamiento global que ubican y explican a los actores y sus trayectorias desde sus posiciones en las estructuras. Es histórica porque tanto en la longue durée del análisis del sistema mundo, como en el enfoque gramsciano de la economía política internacional encontramos una explicación del presente a partir de su inserción en una construcción continua desde los siglos anteriores. Aquí, se enfatiza el despliegue de las hegemonías globales 
desde la escala nacional, las características que asume tal construcción hegemónica al nivel del sistema interestatal y su impacto en la configuración del espacio de lo político.

\section{El enfoque crítico de la economía política internacional}

El estudio de las hegemonías posee un largo recorrido en las relaciones internacionales. Entre los principales enfoques se destacan los realistas y liberales, consolidados dentro de la tradición disciplinar. Sin embargo, desde los años setenta del siglo XX, estas corrientes abrieron importantes debates tanto en su interior como entre ellas, que han resultado en actualizaciones y síntesis relevantes. En el realismo clásico la noción de hegemonía permanecía ligada a una comunidad de Estados con estructura anárquica, moldeable a partir del ejercicio del poder para lograr un tipo de orden que beneficie al Estado hegemónico y, al mismo tiempo, sea tolerable para los Estados subordinados (Carr, 2004; Herrera Santana, 2017). La revisión neorrealista se ha construido y profundizado al interior de este paradigma y, a pesar de sus diferencias, comparte con el enfoque neoliberal una mirada similar sobre la estructura internacional anárquica, con los Estados como actores centrales y el principio de autoayuda como guía para su accionar (Lamy, 2001). El "debate neoneo" que se dio a fines del siglo xx dejó fuera múltiples temas, entre ellos, una revisión más detallada sobre el concepto de hegemonía.

Por el contrario, el giro gramsciano en las relaciones internacionales se construye para ir más allá del limitado alcance de la tendencia mayoritaria (Worth, 2011) y de lo que Cox (2013) denominó "teoría de solución de problemas" (p. 134) de raigambre conservadora (Cohen, 2014, p. 54). El concepto de hegemonía en Cox introduce los componentes gramscianos del consenso y la dirección moral e intelectual de una clase o fracción de clase sobre las demás. Así, la hegemonía se constituye en tres niveles o esferas de acción: 1) la organización de la producción y las fuerzas sociales que surgen de ella, 2) la forma de los Estados y 3) los órdenes mundiales. La interacción no lineal ni jerárquica, sino dialéctica entre los tres niveles o esferas de acción de la hegemonía conforma las "estructuras históricas" (Cox, 1981). Además, dentro de cada uno de estos niveles está contenido un arreglo 


\section{8 / Joel Sidler - Víctor Ramiro Fernández}

particular de condiciones materiales, ideas e instituciones. El nudo de la cuestión es develar qué configuración adoptan las estructuras históricas que dan cuenta de las estabilidades en el sistema internacional y cómo se explican los cambios en ellas.

No obstante, para evitar una teoría cíclica de la historia que explique las estabilidades y los cambios solo a partir de los acoples y desacoples entre los tres niveles en ciertos momentos y lugares, algunos autores recurren al concepto de bloque histórico como momento de equilibrio contradictorio entre las distintas fuerzas sociales. Cox (2013) lo hace mediante una "economía política del mundo" (p. 148) al analizar la interacción entre las tres esferas de acción, mientras que Gill y Law (1989) agregan que todo bloque histórico debe no solo expresar el equilibrio contradictorio entre clases y fracciones de clase al interior de la sociedad civil y sus relaciones de producción, sino también lograr instaurarse a nivel ético-político, lo cual le permite construir y proyectar sus redes políticas y organizacionales.

Por lo tanto, un bloque histórico existe solo a expensas de la existencia de una clase hegemónica, capaz de ser dirigente y dominante al interior de las fronteras de un país ya que "el contexto nacional sigue siendo el único donde se puede fundar un bloque histórico" (Cox, 2016, p. 151). Es en esta escala donde las clases y fracciones de clases disputan la naturaleza de las relaciones de producción y una forma de Estado que las contenga, pero que no las circunscriba solo al interior de sus fronteras. Una vez alcanzado el equilibrio contradictorio que implica un bloque histórico, la clase hegemónica puede traducir sus intereses desde la escala nacional a la internacional; esto es, proyectar las relaciones de producción, las fuerzas sociales y la forma de Estado que mejor se ajusten a sus necesidades. Por ello Cox (2016) afirma que "una hegemonía mundial es, por consiguiente, en sus inicios una expansión hacia el exterior de la hegemonía interna —nacional— establecida por la clase social dominante" (p. 148).

Para alcanzar esa expansión global, la clase hegemónica encuentra en los organismos, instituciones o acuerdos internacionales la capacidad de establecer las reglas necesarias para tal expansión, reducir la 
resistencia de posibles adversarios mediante la absorción de las oposiciones y otorgar legitimidad mediante la incorporación de las élites de los países periféricos (Cox, 1983). Así, la hegemonía opera mediante la construcción de un bloque histórico y el establecimiento de una cohesión social al interior de una forma de Estado-nación y la expansión de un modo de producción a escala global, con central relevancia de las organizaciones internacionales. En ese doble juego, los Estados ocupan un lugar "intermedio, aunque autónomo" (Cox, 2013, p. 148) entre la estructura global de las fuerzas sociales y sus configuraciones locales dentro de cada país.

En consecuencia, para esta corriente de pensamiento la hegemonía global es necesariamente y al mismo tiempo "una estructura social y una estructura política” (Cox, 2016, p. 149), ya que no es solo un ordenamiento jerárquico entre los Estados del concierto internacional, sino que también penetra al interior de cada uno de ellos mediante un modo de producción dominante, que se vincula con otros modelos subordinados y conecta a las clases sociales (Cox, 2016). Por ejemplo, el orden de la segunda posguerra se comprende a partir de la consolidación al interior de Estados Unidos de un bloque histórico que logró conformar un nuevo bloque histórico internacional (Gill \& Law, 1989). Este nuevo orden hegemónico implicó dos transformaciones centrales: la internacionalización del Estado y la internacionalización de la producción (Cox, 1987). La primera se refiere a la orientación de las políticas y prácticas nacionales hacia las exigencias de la economía mundial. Este proceso explica el rol intermedio que ocuparon los Estados nacionales entre los organismos internacionales y los requerimientos de los acuerdos de Bretton Woods, por un lado, y el sostenimiento de la legitimidad política al interior de sus fronteras, por el otro. Esto se observa a partir de la implementación del Plan Marshall, principalmente en Europa Occidental, donde el espacio nacional se configuró como un nuevo ámbito de interacciones sociales, aunque ello no implicó una nueva jerarquización de los demás espacios ni un despliegue homogéneo alrededor del globo (Cox, 1987, p. 253). 
A su vez, la internacionalización de la producción consiste en la creación de una economía mundial de producción internacional, que emerge al interior de la tradicional economía internacional de intercambio (Cox, 1987). Se refiere, por lo tanto, al crecimiento de organizaciones transnacionales de producción, cuyos establecimientos se encuentran en diferentes jurisdicciones territoriales. Ambos procesos de internacionalización habilitaron la construcción de una estructura económica, de seguridad y política centrada en Estados Unidos, con un crecimiento de la demanda agregada global, sostenido por el déficit de su balanza de pagos y una correspondencia de ideas, instituciones y políticas entre las principales naciones capitalistas en lo que Ruggie (1982) denominó "liberalismo arraigado".

Por consiguiente, el ejercicio de la hegemonía estadounidense se constituyó en cada uno de los tres niveles o esferas de acción. Mediante la internacionalización de la producción se aseguró un modelo económico compatible con sus intereses a corto y mediano plazo, a través de la internacionalización del Estado y la construcción de una arquitectura global conformada por los organismos internacionales que le otorgaron una estabilidad temporal a la legitimidad del sistema capitalista. Lo relevante para esta perspectiva es que la internacionalización del Estado, como modificación de la forma de Estado a partir de la constitución de una hegemonía, es un proceso que es disputado en la escala nacional. Como señala Cox (1987), la tendencia hacia la internacionalización del Estado es resistida por grupos sociales domésticos que han quedado en desventaja o han sido excluidos en los nuevos realineamientos (p. 253).

De esta manera, la espacialidad del poder político se reconfigura a partir del accionar hegemónico durante la Pax Americana. En cada espacio nacional alrededor del globo, el ejercicio de la hegemonía estadounidense adoptó características particulares, pero en términos generales la internacionalización de la producción y del Estado se desplegaron en casi todas las latitudes - la excepción fue el espacio de la Unión Soviética-. Ambos procesos constituyeron el preludio para la posterior globalización (Míguez, 2017). 
Ya para los comienzos de la década del setenta, la crisis de la hegemonía estadounidense se hizo presente con la ruptura de los acuerdos de Breton Woods y el abandono del patrón oro. A pesar de ello, los organismos internacionales continuaron como marco institucional para la reconstrucción de un nuevo tipo de estabilidad. Desde entonces, se observa el paso de un bloque hegemónico-internacional a un bloque transnacional que es predominante, es decir, sin hegemonía (Gill, 1995). Este cambio creó las condiciones para, primero, el desarme de las restricciones a la movilidad del capital de la época de posguerra y después para el dominio del capital transnacional. Gill (1995) resume estos cambios en dos direcciones: la expansión del neoliberalismo disciplinar y el neoconstitucionalismo (Bieler \& Morton 2004). Sin embargo, Gill (2008) también destaca que en la medida en que las relaciones sociales capitalistas fueron globalizadas, también la resistencia a ellas logró configurar fuerzas "en movimiento" para oponer al proyecto de globalización neoliberal una globalización "desde abajo" (p. 181). Denomina "príncipe posmoderno" a la nuevas formas de acción política de escala global que se caracterizan por ser diversas, múltiples y no jerárquicas, en contraposición a la figura del partido político moderno, clave para el pensamiento de Gramsci a principios del siglo xx.

En suma, esta primera vertiente permite comprender las hegemonías globales desde su formación en la escala nacional, su constitución en las esferas de las relaciones de producción, las formas de los Estados que la contienen y el orden global que proyectan. Se señaló cómo el propio ejercicio de la hegemonía reconstruye y reconfigura los espacios nacionales a partir de la incorporación de las dinámicas de internacionalización y cómo ello también tiene su correlato en la internacionalización de las resistencias. Así puede comprenderse el

\footnotetext{
1 En clara referencia al "príncipe moderno", figura que Antonio Gramsci recupera del clásico El Príncipe de Nicolás Maquiavelo (1971), publicado por primera vez en 1532. A diferencia de Maquiavelo, Gramsci (1981a) postula la encarnación del poder político ya no en una persona individual, sino en una voluntad colectiva, constituida por el partido político moderno en general y por el Partido Comunista en particular, que debe encargarse de dos puntos fundamentales: la "formación de una voluntad colectiva nacional y popular de la que el moderno príncipe es precisamente la expresión activa y operante, y reforma intelectual y moral" (p. 228).
} 
rol cambiante del Estado en el proceso de reproducción ampliada del capital, la estructuración de relaciones sociales de producción en múltiples escalas y sus consecuencias en el ordenamiento espacial del poder político.

\section{Análisis del sistema mundo}

$\mathrm{Al}$ interior de esta perspectiva también se ubica el análisis del sistema mundo. Este enfoque observa un conjunto de fenómenos políticos y sociales a un nivel sistémico y de larga duración temporal. Las investigaciones de Wallerstein $(1974 ; 1984)$ y Arrighi $(1999 ; 2005 ; 2007)$ son amplias y rigurosas, aquí se retoman sus construcciones en torno al Estado, Estado hegemónico, sistema interestatal y la dimensión espacial. Tales conceptos cobran sentido dentro de la totalidad del sistema mundo capitalista. Wallerstein (1974) señala que el desarrollo de la economía-mundo capitalista es la condición necesaria para la creación de la mayoría de las instituciones o elementos de la actualidad; por ejemplo, las clases sociales, las divisiones étnicas, las nacionalidades y los Estados. Es decir, el sistema mundo es el resultado de las fuerzas económicas que operan a escala mundial, mientras que las clases sociales, nacionalidades y Estados serían el resultado del establecimiento del sistema capitalista en esa escala y no al revés (Wallerstein, 1984).

Wallerstein (1984) define a los Estados como instituciones creadas como reflejo de las necesidades de las fuerzas de clase que operan en la economía-mundo (p. 33). Así, se convierten en un pilar fundamental del sistema ya que son, al mismo tiempo, contenedores de los procesos productivos centrales o periféricos y el reflejo de las fuerzas económicas y de clase que operan en la economía-mundo. Sin embargo, para Wallerstein $(1974 ; 1984)$ son variables dependientes del sistema mundo (Acco, 2018, p. 726), es decir, que sus acciones y características están determinadas por las posiciones que ocupan en la estructura. La economía-mundo capitalista se sirve del sistema interestatal atomizado y en intercompetencia por la movilidad del capital. 
La dimensión espacial ingresa en las relaciones centro-periferia a partir de constatarse un flujo de plusvalor desde los países que concentran actividades periféricas hacia los que concentran actividades centrales. Los últimos tienden a agruparse en unos pocos Estados, mientras que los primeros están diseminados a lo largo de un gran número. Esto deriva en la conceptualización de "Estados centrales" con la capacidad de asegurar cuasimonopolios, y "Estados periféricos" usualmente incapaces de afectar la división del trabajo y forzados a tomar la estructura como dada (Wallerstein, 2004, p. 29). Además, los Estados centrales tienen la capacidad de impulsar cambios en los funcionamientos internos de los demás países, ya sean centrales o periféricos. Así, entran en juego dos elementos cruciales para comprender la dinámica del sistema interestatal desde la perspectiva del sistema mundo: la dominación y la hegemonía. Existen dos maneras para que un Estado alcance el predominio. La primera es transformar la economía-mundo en un imperiomundo, con una única autoridad política. Esto nunca ha sucedido en el sistema mundo moderno. La segunda es obtener la hegemonía en el sistema mundo. Solo tres poderes han alcanzado la hegemonía desde el establecimiento de la economía-mundo capitalista y por periodos relativamente breves. Primero las Provincias Unidas, a mediados del siglo XVII, luego el Reino Unido a mediados del siglo XIX y Estados Unidos desde el fin de la segunda posguerra.

Los Estados hegemónicos son, según Wallerstein (2004, pp. 57-58), aquellos que durante un cierto periodo lograron establecer las reglas de juego en el sistema interestatal, dominar la economía-mundo (en términos de producción, comercio y finanzas), obtener ventajas políticas con un mínimo uso de la fuerza militar (que sin embargo poseen en gran cantidad) y formular el lenguaje cultural con el que se discute el mundo. Un periodo de hegemonía crea, según esta perspectiva, un tipo de estabilidad dentro de la cual las empresas se ven favorecidas, especialmente las ligadas al poder hegemónico y con posiciones monopólicas. Sin embargo, los periodos hegemónicos son pocos y breves. El poderío económico y militar de los Estados hegemónicos tiende a disminuir frente a las mejoras de otros Estados, con ello decrece su poder político y se acrecienta la necesidad de recurrir a la fuerza militar. 
Arrighi (1999), por su parte, señala que los Estados hegemónicos son actores centrales en los procesos de expansión material y financiera. Ello implica que el movimiento de formas de capital unidas mayormente a la producción o a la financiarización marcan el ritmo de los ciclos sistémicos de acumulación (CSA). Cada CSA se caracteriza por una fase de expansión material y una fase de expansión financiera y sus despliegues dan la pauta de comportamiento del sistema capitalista a escala global, así como de las trayectorias de los Estados hegemónicos. Además, es en el interregno entre el desarrollo y la finalización de una fase financiera y el comienzo y despliegue de una nueva fase material donde los traspasos hegemónicos generalmente ocurren (Arrighi, 2005).

El poder hegemónico es, para Arrighi (2005), algo más y diferente que el dominio puro y simple; además, define a la hegemonía como ese poder adicional que habilita el ejercicio de un liderazgo intelectual y moral. De esta manera, la hegemonía refiere al poder de un Estado para ejercer funciones de liderazgo y gobierno sobre un sistema de Estados soberanos. Esta definición agrega un elemento central a la acuñada por Wallerstein (1974; 1984; 2004). Como se señaló, para Wallerstein (1974; 1984; 2004) la hegemonía se expresaría en la capacidad de establecer las reglas de funcionamiento en el sistema interestatal, mientras que Arrighi (1999; 2004; 2007) agrega que esas reglas no expresan solo el beneficio del hegemón, sino que deben también incluir un consenso por parte de los Estados subordinados, a la forma de una prosecución del interés general. Este último carácter refuerza el giro gramsciano de la perspectiva del sistema mundo y señala puntos de conexión con la economía política internacional.

En resumen, en la esfera global la búsqueda del interés general implica la capacidad de proyectarse como modelo a seguir para atraer a los demás Estados hacia su forma de acumulación de capital y control territorial, formando un imaginario capaz de presentar al Estado hegemónico como "faro" del sistema interestatal. En palabras de Arrighi (1999): "un Estado puede convertirse en una potencia hegemónica mundial porque puede afirmar de modo verosímil que la expansión 
de su poder respecto a algunos o incluso todos los demás Estados es de interés general" (p. 45).

En consecuencia, observado en su lógica sistémica y en la longue durée, el moderno sistema mundo adopta una naturaleza evolutiva y contradictoria, en cuyo interior las hegemonías ocupan un papel central en la configuración y transformación del sistema a lo largo de los CSA. Un aspecto relevante en esta dinámica es el intento de resolver la contradicción entre la incesante acumulación de capital, por un lado, y la organización del espacio político, por el otro. En términos de Arrighi (2005), ello se presenta mediante una oposición entre las lógicas del capital y las lógicas territoriales. En las primeras, las adquisiciones territoriales son un medio en la búsqueda por acumular capital, mientras que en las segundas, la extensión territorial y la densidad poblacional se encuentran identificadas con el poder, y la acumulación de capital es un medio para mantener y expandir el poder territorial.

En términos históricos, ambas han construido estrategias opuestas para la formación de Estados. Esta contradicción es una característica intrínseca del sistema interestatal, donde se ponen en juego los flujos de capital y la espacialidad donde los gobiernos ejercen su poder. Generalmente, la resolución se canaliza mediante la reorganización del espacio económico-político mundial, a partir de la acción de liderazgo desempeñada en cada momento histórico por el Estado hegemónico correspondiente. Así, Arrighi (2005) analiza la crisis de la hegemonía estadounidense y recupera lo que David Harvey (2005) denominó spatial fix para dar cuenta de la demora temporal y la expansión geográfica como respuestas frente a las crisis de sobreacumulación capitalista.

Desde este enfoque, a mediano plazo la producción del espacio por parte del sistema capitalista puede llevar al desarrollo de nuevas regiones que impliquen una significativa competencia para el centro hegemónico, lo que resulta en una devaluación de su poder y una amenaza a la estabilidad. Esto permite observar la crisis de los años setenta y la intensificación de la competencia interestatal como la 
"crisis señal" de la hegemonía estadounidense (Arrighi, 1999; Harvey, 1998) y el inicio de la consolidación del este asiático, primero, y China, después, como el nuevo centro de acumulación material a escala global (Fernández \& Moretti, 2020).

En síntesis, tanto el análisis del sistema mundo como la vertiente crítica de la economía política internacional permiten comprender el funcionamiento sistémico e histórico de las dinámicas del sistema capitalista. Se ha puesto énfasis en presentar, para cada una, el rol de los Estados en la estructura global y, en especial, de los Estados hegemónicos y su función de liderazgo frente al conjunto de Estados. En estos abordajes, los tres elementos (Estados, espacios y hegemonías) presentan un tipo particular de retroalimentación conceptual.

En la primera vertiente señalada, en especial en la propuesta de Cox $(1981 ; 1983 ; 1987 ; 2013 ; 2016)$, la escala nacional se expresa como el lugar donde la hegemonía tiene su comienzo a partir del éxito de una clase o fracción de clase en la presentación de sus intereses como parte del interés general y en el establecimiento de relaciones de producción y formas de Estado que los sostengan y reproduzcan. Desde allí, es capaz de instalarse globalmente en la construcción supranacionalinstitucional y desplegar los discursos y prácticas que expresan una hegemonía espacial global, con anclaje en el bloque histórico y su clase hegemónica. Frente a ello, el análisis del sistema mundo pone el énfasis en el capital y la formación interestatal por ciclos con relevos de agencias estatales y nuevas formas de acumulación y posicionamiento espacial. Si bien la mirada sistémica subordina el rol de los Estados a las lógicas cíclicas y en ello las nacionalidades son un subproducto del sistema mundo capitalista, el rol de los Estados es protagónico al momento de intervenir en el comercio desigual y el desenvolvimiento de las lógicas territoriales y capitalistas en cada momento; es en su accionar donde se centra el ejercicio hegemónico a escala global.

No obstante las especificidades dentro de y entre ellas, ambas corrientes confluyen en el reconocimiento de un sistema interestatal que se forma a partir de Estados con un control territorial desde el que se configuran 
históricamente proyectos hegemónicos que logran un orden - transitorio—global. Además, ambas construyen desde una fusión teórica la correspondencia entre Estado y liderazgo hegemónico con el despliegue cíclico-sistémico en el que el vínculo de las lógicas e intereses estatales y del capital, con sus especificidades y realimentaciones, reconocen la creciente presencia de una configuración supranacional bajo control de los Estados hegemónicos. En esta instancia supranacional se forma el dispositivo institucional de discursos, prácticas o imaginarios que se difunden e implementan mediante la participación subalterna de los Estados y espacios no hegemónicos.

\section{Parte 2: Perspectiva posestructural relacional}

La recuperación de los aportes de la geografía política en torno a la relevancia del espacio ${ }^{2}$ y su transformación permite dar cuenta de una dinámica de interacciones organizada a partir de redes con distintos actores. Las contribuciones de John Agnew (1994; 2005) en la indagación y revisión espacial del concepto de hegemonía encuentran complemento en los trabajos de John Allen (2003) y Saskia Sassen (2013), en relación con las nociones de territorialización y espacio político junto a sus análisis de las transformaciones en la soberanía de los Estados (Sassen, 2008).

Los argumentos al interior de esta parte forman una perspectiva posestructural porque elaboran un conjunto de nociones que desplazan las interpretaciones sistémico-estructurales para poner énfasis en las diversidades, contingencias y multiplicidades que emergen al observar las configuraciones estatales a escala global. Ello lleva a redefiniciones en torno a la territorialidad, las fronteras y la soberanía que rompen con los postulados clásicos aceptados y divulgados desde el Tratado de Westfalia en adelante. Ello abona a perspectivas planteadas en el campo de las relaciones internacionales por autores como Krasner

\footnotetext{
2 Para los efectos de este artículo, se recupera la definición de Agnew (1994), quien entiende al espacio como aquello donde los procesos político-económicos tienen lugar y sus efectos sobre tales procesos (p. 55).
} 
(2001), para quien el atributo de la soberanía estatal ha sido tomado como dado e inmutable a lo largo del tiempo por parte del neorealismo y del neoliberalismo institucional, donde se asume a los Estados como actores racionales, unitarios e independientes. Esto ha derivado en enfoques que han sido incapaces de analizar las transformaciones recientes en torno al funcionamiento de los Estados y la espacialidad del poder político.

Un aspecto de esta perspectiva posestructural, es su componente relacional, que sitúa el poder en el campo de la interactividad entre diversos agentes, es decir, no solo entre las instituciones o en el ejercicio de las administraciones centrales de un Estado, sino en las capacidades de múltiples entidades de disolver, alterar y reconstruir fronteras. Así, entran en juego las espacialidades globales, observadas con un nuevo lente metodológico, donde las fronteras estatales son asumidas menos como rígidas y más como cambiantes. Los diversos tipos de flujos económicos, poblacionales, culturales e informacionales son responsables de una virtual disolución de las barreras estatales, que crean nuevos y alternativos imaginarios en torno a la soberanía territorial, por largo tiempo tomada como dada por los Estados-nación.

\section{Hegemonía y nuevas geografías de poder}

Buena parte del campo teórico, influido por la impronta weberiana, asumió el Estado como una entidad territorial fija, que ha funcionado de forma más o menos similar a lo largo del tiempo (Agnew, 1994). Sin embargo, en las últimas décadas se ha problematizado esta asunción y señalado que, a pesar de que las normas establecidas por los Estados posean un carácter territorial definido, esta territorialidad ha atravesado transformaciones en cada momento histórico a causa de la dinámica geopolítica. Agnew (2003) sostiene que desde la década de los setenta un nuevo mundo posmoderno ha emergido. En este, las reglas que establecieron la organización del espacio a partir de las capacidades estatales para contener de forma territorial los efectos externos se están desmoronando. Caracterizado por la rápida movilidad del capital, la centralidad de las tecnologías de la información y el crecimiento de las conexiones en red entre diversas ciudades-regiones del mundo, el 
espacio se encuentra reconfigurado. Esto conlleva un replanteo de los conocimientos establecidos sobre el ejercicio del poder por parte de las autoridades políticas y evaluar cómo esto afecta el accionar de la hegemonía (Agnew, 2003).

Analizar el poder político solo como territorial dificulta la comprensión de las metamorfosis actuales. Como señala Agnew (1994), la mayoría de los enfoques comparten con frecuencia un conjunto de supuestos geográficos que otorgan preponderancia a la forma territorial de comprender al Estado: 1) reificar los territorios de los Estados como unidades soberanas fijas, lo que lleva a deshistorizar los procesos de formación estatal y a tomarlos por dados; 2) utilizar la dicotomía nacional/ internacional (o domestic/foreing en inglés) para dar cuenta de procesos económicos y políticos interconectados, en diferentes escalas, lo que ha resultado en simplificaciones que conducen a errores teóricos, y 3) anteponer la existencia del Estado como contenedor de la sociedad, es decir, la nacionalización de la sociedad.

Con la crítica y superación de estos supuestos, la construcción teórica en torno a los procesos de hegemonía adquiere una connotación diferente respecto a lo presentado en la perspectiva anterior. Mientras en aquella, en especial para el sistema mundo, el hegemón es necesariamente un Estado, ya que solo la combinación de preeminencia económica, política y militar proporciona suficiente poder y autoridad para pacificar y dirigir el sistema mundo capitalista (Parnreiter, 2018, p. 5)

En este sentido, Agnew (2005) difiere del sistema mundo y las visiones cíclicas de los procesos hegemónicos. Este autor considera que aquella forma de acercarse a la geopolítica del sistema capitalista otorga demasiada atención a los roles de determinados Estados "clave" así como a las "identidades" de quién o qué ejerce la hegemonía en detrimento del carácter distintivo del propio ejercicio de ella (p. 58). Así, según Agnew (2005), la hegemonía estadounidense durante la segunda mitad del siglo xx puede explicarse mejor a partir de una consideración que escape de la trampa territorial (territorial trap). Para ello, le otorga una especial relevancia al lugar que ejerce la hegemonía y al desarrollo histórico de un conjunto de características sociales y culturales que, 


\section{0 / Joel Sidler - Víctor Ramiro Fernández}

para finales del siglo XIX, diferenciaron a Estados Unidos de otras sociedades nacionales capitalistas.

De esta manera, la hegemonía estadounidense es la conjunción de su formación histórica, que comprende las particularidades de una sociedad de mercado, junto con la creación de una economía global mediante los organismos internacionales de posguerra capaces de generar el consenso a su ejercicio del poder. Este argumento tiene un punto de contacto con los procesos de internacionalización descritos en la perspectiva anterior. Tanto la internacionalización del Estado, como del capital son elementos centrales de un tipo de proyección hegemónica que implicó un cambio abrupto en la geografía del poder, pasando de una estrictamente territorial hacia una funcional y relacional forma de espacialidad (Agnew, 2005). Ello produjo alteraciones en las reglas de interacción espacial en términos comerciales y de flujos de capital, que en las décadas posteriores la globalización usó como plataforma de despegue. Siguiendo a Agnew (1994), la globalización fue el resultado necesario de la hegemonía global estadounidense y representa una brusca aceleración, con reformulación geográfica, de la progresiva universalización de los procesos de mercantilización y acumulación capitalista. Desde entonces, tanto los Estados en su forma territorial como los actores no territoriales operan en un mundo en el cual las fronteras son cada vez más permeables y sujetas al accionar de diversas redes de poder y flujos económicos, que no necesariamente responden a una singular representación territorial del espacio (Agnew, 1994, p. 72). Si bien tal forma territorial no desaparece, el mundo que emerge a finales del siglo XX requiere de herramientas conceptuales capaces de evitar la trampa territorial.

Entre esas herramientas se ubican los trabajos de Sassen (2013), quien sostiene que las jaulas de la autoridad territorial nacional se están rompiendo (p. 30). Desde su mirada, las fronteras se configuran como lugares de intervención con diversas lógicas geográficas e institucionales, donde interactúan flujos de capital, información y personas. Para Sassen (2009; 2013), el dominio del Estado-nación ha simplificado el concepto "frontera". Sin embargo, desde finales del 
siglo xx la globalización ha demostrado que las fronteras no necesariamente siguen los estándares geográficos, sino que pueden extenderse y funcionar hacia el interior del territorio nacional (Sassen, 2009).

La autora se enfoca en novedosos tipos de bordering capabilities, ${ }^{3}$ que dan forma a espacios con fronteras que funcionan de manera transversal a las estatales. Estos espacios se distinguen de los flujos tradicionales - generalmente regulados a nivel nacional por los Estados- por implicar un movimiento al interior de la territorialidad estatal, creando nuevos espacios y nuevas fronteras internas. De esta manera, el sistema interestatal de Estados soberanos como unidades estructurales comienza a transformarse y el significado de "soberanía" en su formato westfaliano cambia; si bien se mantiene como una propiedad sistémica, el proceso que intenta conceptualizar ya no existe como tal (Sassen, 2009).

Lo particular de los nuevos tipos de capacidades de fronterización es que logran amalgamar la presencia de lo global y lo local dentro de lo nacional y crear múltiples territorios transversales a las fronteras tradicionales, que funcionan como ensamblajes globalmente escalados que incluyen componentes subnacionales, nacionales y supranacionales (Sassen 2009, p. 570). Ello conlleva a crecientes tensiones alrededor de cómo ingresa y adquiere lugar la hegemonía y el rol de los Estados en estas nuevas dinámicas de ensamblajes que son, al mismo tiempo, globales, nacionales y locales.

Estos nuevos ensamblajes han transformado las lógicas organizativas, esto se refiere al paso desde la institucionalización de un perímetro determinado para un territorio determinado, hacia las múltiples y transversales "fronteras" que se construyen a través de ellos. Según la autora, las incipientes formaciones de nuevas capacidades de fron-

\footnotetext{
3 Estas dinámicas de construcción de fronteras se despliegan de forma independiente a las fronteras estatales, no las reemplazan sino que cambian las lógicas de las capacidades de fronterización ya que producen una variedad de nuevas fronteras al interior del territorio nacional, lo que implica un cambio en los imaginarios y prácticas tradicionales en torno a la soberanía territorial (Sassen, 2009, p. 3).
} 


\section{I Joel Sidler - Víctor Ramiro Fernández}

terización y prácticas estatales llevan a una desnacionalización parcial y afectan la comprensión tradicional sobre lo nacional y sus fronteras (Sassen, 2009). En este sentido, Glassman (1999) señala, por ejemplo, que la internacionalización del Estado pone de manifiesto estos cambios a partir de que el aparato del Estado se vuelve mayormente orientado a facilitar la acumulación del capital para los inversores más internacionalizados, independientemente de su nacionalidad (p. 673).

Otra contribución significativa es la de Jim Buller (2018), quien sostiene que la arraigada presencia de la trampa territorial en las investigaciones de ciencia política y relaciones internacionales arroja una visión limitada del poder estatal. Frente a las transformaciones externas y los desafíos globales, estas exploraciones construyen una representación del Estado como obsoleto u obstinado (Hoffmann, 1966). Por el contrario, Buller (2018) argumenta que los Estados poseen una significativa presencia en el espacio transnacional si este es observado funcionalmente. Desde esta óptica, los actores estatales conservan capacidades para moldear e incluso comandar el espacio mediante diversas fusiones de objetos "internos" e "internacionales", a las cuales denomina casos de gobernanza conjunta (linkage governance). Estos consisten en conjuntos de instrumentos de políticas públicas diseñados para gestionar o administrar el espacio desde una lógica funcional junto a las redes transnacionales que operan en este. Mediante tales instrumentos, los actores estatales logran combinar anteriormente separadas instituciones nacionales e internacionales.

Si se retoman los argumentos de Sassen $(2008 ; 2009$; 2013; 2019) y Buller (2018) a la luz de una concepción del poder como un efecto relacional de la interacción social, que es inherentemente espacial y viceversa, donde la espacialidad está imbuida de poder (Allen, 2003), los ensamblajes, las capacidades de fronterización y las combinaciones de gobernanzas conjuntas se presentan como conceptualizaciones que pretenden dar cuenta de surgimientos de nuevos tipos de territorialización del espacio político, con formas de poder que emergen de ellas y de las que están impregnadas. De esta manera, si bien deshacen los supuestos clásicos en torno a la soberanía de los 
Estados, no erigen nuevos en relación a la mecanicidad de los procesos globalizadores. Por el contrario, estos abordajes dan cuenta de artificios novedosos, tecnologías de gobierno estatales y no estatales que surgen de las interrelaciones entre diversos actores que a veces enfrentan y otras enhebran diversos intereses. Así, se da una complejización en la relación entre poder político, espacio y Estado de manera que, por ejemplo, los cambios en las soberanías de los Estados-nación no necesariamente se explican como pérdidas de poder en términos regulatorios a raíz de presiones externas, sino, por el contrario, como novedosas expresiones de las capacidades para desnacionalizar, privatizar y seguir agendas globales que históricamente han sido propias de los Estados (Sassen, 2008).

En suma, estos aportes, con sus particularidades y diferencias, comparten una perspectiva que mejora la comprensión del giro en las geografías del poder que acompañó el despliegue de la hegemonía estadounidense. Esta se caracterizó por una construcción espacial alejada de la estricta territorialidad del Estado-nación más cercana a una lógica funcional y relacional del espacio. Intentar abordarla desde las concepciones tradicionales deja de lado las especificidades del ejercicio hegemónico y no revela su impacto en las transformaciones presentes. Con la relativización de la escala nacional como el lugar original del ejercicio del poder político, surgen nuevas dinámicas entre los actores territoriales y no territoriales, y las fronteras abandonan su carácter fijo y estable para asumir una transformación y complejización de su relevancia y efectos en el escenario político global.

El proceso de globalización y las nuevas formas de territorialización no implican solo novedades en las formas de ocupar el espacio, sino que producen y llevan intrínsecas nuevas formas de poder que son diferentes de las previamente implicadas en el ejercicio territorial del Estado-nación. Así, el despliegue del poder político se ejerce en nuevas geografías, con nuevos actores y nuevas dinámicas que ponen en cuestión supuestas capacidades de autolimitación territorial en la acción política, arraigadas en la impronta weberiana de buena parte de la academia. Dar cuenta de los cambios configurativos permite comprender los emergentes imaginarios alrededor del ejercicio de la hegemonía a 
nivel global, los múltiples factores involucrados en el deterioro de la estadounidense y los sucesos actuales que ubican a las ciudadesglobales y las regiones sub- y macronacionales como espacios relevantes para comprender los procesos geopolíticos y geoeconómicos.

\section{Parte 3: Contribuciones para una perspectiva estructural histórica y dinámica}

Las perspectivas sistémico-estructural histórica y posestructural relacional contienen elementos relevantes para dar cuenta de los desafíos actuales que enfrentan los Estados en general y los espacios periféricos en particular. Su complementación —antes que su colisiónpermite construir una perspectiva capaz de interpelar la complejidad de los procesos políticos y económicos que involucran la crisis de la hegemonía estadounidense y las respuestas estatales y espaciales en América Latina durante el presente siglo. Dicha complementariedad promueve un análisis que invita a abordar los procesos actuales evitando confrontar los niveles macro o micro, así como articular un abordaje de larga duración con uno focalizado en "la historia reciente". La observación integral de esos procesos requiere comprenderlos a partir de sus interrelaciones con diversas escalas que, lejos de estar jerárquicamente establecidas, se imbrican y funcionan de maneras transversales (Sassen, 2019). Así, la producción de los macroprocesos se alimenta de y se comprende desde las trayectorias específicas de cada espacio (Tilly, 2016), capitalizándolas y combinándolas con las particularidades que asume su actual presencia.

Las formas en que procesos de larga duración conviven y se recrean en contextos recientes y en espacios específicos no solo permiten reconocer la construcción de diferentes estructuras - económicas, institucionales - sino sus más actuales transformaciones a partir de un complejo campo de factores contingentes que le dan nuevas formas y contenidos. En este sentido, las perspectivas presentadas en las primeras dos partes, más allá de sus tensiones tanto al interior de cada una como entre ellas, abonan elementos que permiten síntesis relevantes. Por una parte, el enfoque crítico de la economía política 
internacional se entronca con el análisis del sistema mundo en una mirada global que hace ingresar con tintes gramscianos el papel de las ideas y el poder en la conformación de un hegemón que es cíclicamente variable. Sin embargo, esta mirada de larga duración no puede quedar solo orientada a los espacios centrales, sino que debe ser complementada con una observación detallada sobre la forma en que aspectos estructurales e históricos se combinan con las contingencias y sus renovados flujos en los centros y las periferias.

Esto resulta clave para comprender las formas de existencia y ejercicio que asumen las hegemonías a nivel global, los cambios en estas y sus diferenciales efectos en los distintos espacios, y su imbricación con las particularidades propias al interior de cada uno de ellos. No menos importante, esto permite observar cómo los cambios en los espacios periféricos afectan el propio proceso global. Para ello, es crucial profundizar en el gran desafío asumido por una parte de la economía política internacional de trascender el análisis del norte global y comprender el funcionamiento y la relevancia de las regiones, asimilando y reactualizando los propios insumos conceptuales provenientes de ella (Tussie \& Riggirozzi, 2015). En esa tarea, América Latina realizó un cúmulo temprano de aportes que conforman aun hoy conceptos principales en el campo de las relaciones internacionales. Entre tales aportes se encuentran los que tanto el estructuralismo latinoamericano (Prebisch, 1986) como el dependentismo (Cardoso \& Faletto, 1977; Dos Santos, 1978), con sus peculiaridades, realizaron para entender la región como parte de un sistema que configura históricamente estructuras centro-periferia, a partir de una formación y relacionamiento socioeconómico-espacial desigual. Bajo el tópico del "desarrollo" como aglutinante, desde esas contribuciones se gestaron diagnósticos, debates y propuestas que hicieron visibles las formas en que los espacios nacionales y la región se integraron al proceso de gobernanza hegemónica (Deciancio, 2018).

Por otra parte, lejos de los determinismos y las lógicas inmanentes, la perspectiva posestructural relacional abre un conducto interpretativo para trabajar en las porosidades del espacio. Con ella es posible 


\section{6 / Joel Sidler - Víctor Ramiro Fernández}

observar los múltiples flujos que penetran los espacios delimitadoslimitados (bounded spaces) y formatean variable y contingentemente los imaginarios para adaptarlos a nuevos procesos y nuevas formas de construcción de poder, que incluyen imprevisibles y contingentes circuitos de prácticas (Gibson Graham, 1996). Esto se vuelve relevante si se considera que sobre esos imaginarios y sus bases materiales — que transcurren en forma de flujos, como el financiamiento, por ejemplo- se configuran los dispositivos de acción estatal e institucional. Así, se establecen tecnologías de gobierno (casos de gobernanzas conjuntas) donde el poder circula y se alteran o cristalizan formas de subordinación. Bajo tales dispositivos, los Estados y sus distintas escalas son ubicados de manera subordinada o dominante dentro del sistema interestatal.

En la propuesta de complementación entre grandes estructuras y aquellas más delimitadas — históricamente formadas - entre los macro procesos (Tilly, 1984) y los espacios de flujos que interactúan con y a través de esas estructuras, resulta fundamental considerar cómo, en el escenario global de la nueva centuria, se alteran las jerarquías y formas operativas del sistema mundo y su dinámica hegemónica. En dicha alteración, se modifican los modos en que operaron y fueron concebidas las estructuras y relaciones centro-periferia durante el siglo Xx por el estructuralismo y las teorías de la dependencia, lo que marca múltiples desafíos a escenarios periféricos como el latinoamericano. La complementariedad permite, en este caso, observar cómo las estructuras organizacionales que cristalizaron las formas de existencia y las relaciones centro-periferia no son disueltas sino reutilizadas y redefinidas a partir de un nuevo complejo relacional, donde una multiplicidad de flujos y dispositivos no solo financieros sino también conceptuales formatean el nuevo campo de acción geoeconómico y geopolítico, apelando a renovados imaginarios y relaciones materiales.

Esta perspectiva asentada en la complementariedad deja lugar para profundizar en las posibilidades de movilidad que tienen los actores y espacios que habitan las estructuras resultantes de esas trayectorias y cómo encuentran distintos condicionamientos que son históricamente 
variables. Esto es relevante ya que logra contextualizar las dinámicas geopolíticas y geoeconómicas dentro de los procesos de larga duración en los que se forjaron estructuras y se demarcaron espacios de competencia y formaciones estatales con disímiles capacidades para el ejercicio político tanto sobre territorios que reclaman como propios, como para el desempeño en las dimensiones no estatalmente territorializadas o, en otros términos, en los procesos que desbordan la territorialidad.

Por lo tanto, una perspectiva estructural, histórica y dinámica pretende ser capaz de capturar ese doble juego: dar cuenta de la posición sistémica de las construcciones estatales, atendiendo también a sus interacciones novedosas como formas de rearticulación a nivel global. De esta manera, lo cíclico del sistema capitalista y lo estructural y lo jerárquico del sistema interestatal interactúan de una manera no determinante sino realimentaria con las trayectorias estatales y las especificidades nacional-regionales que emergen de novedosas interacciones espaciales. De este intercambio se originan coconstrucciones que son históricas y dinámicas a causa de la combinación del proceso de construcción estatal a lo largo del tiempo, con las respuestas a los procesos cíclicos enmarcadas en las particularidades de cada espacio. De esta manera es posible observar el papel de las configuraciones estatales centrales y periféricas en el despliegue de y en el involucramiento en las hegemonías cíclicas y las reconversiones que en ellas opera el capital productivo y financiero en diferentes bloques históricos. Desde allí, es posible evitar una teoría de la determinación y explorar espacios de autonomías estatales dentro un funcionamiento sistémico (Jaguaribe, 1979).

En consecuencia, los órdenes globales en general y los órdenes hegemónicos en particular pueden ser comprendidos ya no como elementos estructurales o derivas verticales del funcionamiento sistémico, sino como resultados cambiantes e inestables a causa de la intervención entrelazada de múltiples escalas (Ikenberry \& Nexon, 2019). De ello dan cuenta los aportes de la perspectiva posestructural relacional, desde la cual las territorialidades se asumen no como inmunidades perennes, sino como espacios reelaborados y penetrados, pero también como plataformas con diferentes capacidades 


\section{8 / Joel Sidler - Víctor Ramiro Fernández}

de acción y respuesta a los desafíos de la competencia interestatal como a las imposiciones del capital y sus flujos (Fernández, 2017). En medio de esto, el ejercicio de la hegemonía se vuelve un elemento central en la construcción de imaginarios globales en torno a lo posible y lo deseable en la práctica política, el espacio de intervención y los actores legitimados para la toma de decisiones. Así, lo relevante para nuestra posición es dar forma a una herramienta analítica que nos permita comprender cómo ingresa la dinámica sistémica en una interacción conflictiva entre los flujos y las estatidades que los reciben, las configuraciones territoriales que toman relevancia junto a sus capacidades para performar los flujos, de ser performadas por ellos y de configurar imaginarios capaces de devenir hegemónicos.

En otras palabras, los flujos ingresan sobre territorialidades particulares, su accionar cuestiona la dominación Estado-céntrica de los territorios y penetran estatidades que poseen diversas capacidades de reaccionar a ellos, orientarlos, dirigirlos y gobernarlos. Esas capacidades ya no están concentradas en actores puramente estatales de la escala nacional, sino distribuidas en múltiples y diferenciados agentes locales, regionales y globales. En este punto se introduce la pregunta por las autonomías nacional-regionales posibles frente al despliegue de la hegemonía a nivel global; más aún, por las instancias de autonomía en las regiones periféricas del sistema mundo, el rol y los desafíos para las estatidades nacionales que, transformadas, no desaparecen de la toma de decisiones.

Para atender estas cuestiones, las perspectivas presentadas en las primeras dos partes tienden a una aproximación metodológica donde prima el examen de la dinámica global en perjuicio del reconocimiento de la forma como las trayectorias nacionales y regionales, formadas históricamente, interaccionan con los procesos globales y cíclicos. Aquí se resalta que esas trayectorias dan como resultado estatalidades diferenciales en términos de autonomía y capacidad para responder a estos procesos, que resultan tanto de la competencia interestatal y el realineamiento espacial de la lógica del capital, como lo reconoce la perspectiva sistémico estructural histórica, como de la constante renovación de los flujos materiales e inmateriales que intervienen en 
la rehechura de esa competencia y ese realineamiento desde una profunda redefinición de las fronteras del poder político y económico, señalado por la perspectiva posestructural relacional.

Por consiguiente, recuperar las trayectorias históricas particulares mediante la combinación de ambas perspectivas permite comprender cómo sociedades y Estados se conforman desde una interacción variable con las estructuras y lógicas sistémicas y los múltiples y acelerados flujos externos (culturales, simbólicos y financieros). Sin embargo, resulta fundamental el aporte de teorías latinoamericanas que resaltan el reconocimiento de las posiciones y capacidades que poseen los diferentes espacios periféricos y sus actores para enfrentar los procesos globales. En definitiva, para orientar una investigación sobre las autonomías posibles en los espacios latinoamericanos y las capacidades de sus Estados para responder a las tendencias globales o incluso para construir ejercicios contrahegemónicos, es necesaria una complementación creativa de las dos perspectivas presentadas.

El carácter estructural, histórico y dinámico que combina la perspectiva propuesta reconoce el posicionamiento periférico y propone retomarlo como un escenario que, siempre condicionado, incluye en su interior diferentes Estados con variables capacidades para formular respuestas a la reconfiguración dialéctica tanto de las estructuras sistémicas como de las dinámicas y flujos que rehacen esas estructuras, reelaboran la organización estatal y rediseñan los imaginarios bajo los cuales se disputan los proyectos hegemónicos. De esta manera, las estructuras y los flujos no solo conviven y se solapan, sino que modifican las plataformas institucionales y lógicas de funcionamiento que operan sobre los Estados y conforman y ejercen los imaginarios y acciones que dan lugar al despliegue de hegemonías y al reordenamiento del espacio. Esta perspectiva pretende ser una herramienta para captar esa dinámica entre flujos y estructuras. 


\section{Conclusiones: Desafíos y oportunidades para una nueva estatidad en la periferia}

En este artículo se presentaron dos perspectivas que se nutren de diversos aportes, tensionan entre sí al tiempo que muestran discrepancias a su interior. La primera de ellas, la perspectiva sistémicoestructural histórica, contiene una corriente ligada a la economía política internacional crítica y otra centrada en el análisis del sistema mundo. La segunda, la perspectiva posestructural relacional, recupera una variedad de investigaciones en torno a las nuevas dinámicas de territorialización del poder político, la desnacionalización de las fronteras y los dispositivos que accionan y construyen nuevos ensamblajes. De ambas se extrajeron elementos relevantes para formular una propuesta analítica sustentada, en buena medida, en capitalizar y complementar las anteriores, procurando identificar y afrontar los desafíos y las oportunidades que se presentan para las estatidades en los espacios periféricos en el contexto actual. Se planteó una vindicación de la estatalidad comprendida desde un funcionamiento estructural, que se combina actualmente con la transformación del ciclo sistémico de acumulación, incorporando los insumos analíticos posestructurales que resaltan la movilidad espacial y las tensiones creadas entre las fronteras.

El argumento desplegado buscó considerar la relevancia de observar cómo las macro estructuras globales interactúan con las configuraciones que emergen de los espacios nacionales y regionales periféricos, así como el modo en que estructuras y flujos que actúan en estos espacios se redefinen mutuamente. Esa complementariedad permite abordar con mayor precisión cómo y por qué los Estados latinoamericanos enfrentan de manera diferencial la dinámica centroperiferia y exhiben particulares fortalezas o debilidades, construidas históricamente, para responder de forma alternativa. Así, se destacó la centralidad actual del accionar de las redes políticas y económicas que interpelan e ingresan al Estado y cómo este, a su vez, en forma diferencial a partir de sus trayectorias configurativas, también les da forma a esas redes y las fuerzas transnacionales y rehace coaliciones con actores locales y no locales. 
La perspectiva estructural, histórica y dinámica surge de esa complementariedad y procura, por un lado, otorgarle un lugar relevante al análisis de la larga duración, que abona a reconocer la formación de espacios estructuralmente delimitados en los cuales las estatidades se integran dentro de una lógica sistémica. En esta última, Estados nacionales y organizaciones supranacionales configuran trayectorias asimétricas y vínculos en conflicto que han estructurado y reestructurado al capitalismo en su dinámica histórica, formando parte de un sistema interestatal donde se edifica un poder hegemónico. Sin embargo, esta perspectiva reconoce que esas relaciones se vieron transformadas durante el último cuarto del siglo xx y los comienzos del XXI a partir del ingreso y la creciente relevancia de dinámicas desterritorializadas. Por ello, sin recaer en la disolución de las estructuras estatales por los flujos, se les reconoce un papel fundamental para reconfigurar las propias bases sociales, los imaginarios y las dinámicas interestatales bajo las que se redefine ese poder hegemónico.

Una perspectiva estructural, histórica y dinámica puede comenzar a construir el conjunto de herramientas conceptuales para captar esa lógica interrelacional entre flujos y estructuras. Puede ser útil para dar cuenta de las capacidades de respuesta de los espacios periféricos, así como de las posibilidades reales y potenciales de establecer contralógicas y proyectos alternativos, fundados en determinados espacios y márgenes de acción que, resultado de múltiples contingencias y trayectorias históricas, se hacen lugar dentro de los reacomodamientos cíclicos y la redefinición de las estructuras históricamente conformadas.

Estos atienden a la coproducción de dinámicas que son, al mismo tiempo, internas y externas, formadas desde estructuras y recreadas desde múltiples flujos con poder. La complementariedad propuesta aporta una base conceptual y metodológica que abre compuertas para el análisis de las dinámicas globales más recientes como el ascenso del Sur Global, la crisis de la hegemonía de Estados Unidos y el regionalismo poshegemónico. 


\section{Referencias}

Acco, M. A. (2018). Os Estados, o sistema-mundo capitalista e o sistema interestatal: uma leitura crítica das contribuições de Immanuel Wallerstein. Brazilian Journal of PoliticalEconomy, 38(4), 708-730. https://dx.doi. org/10.1590/0101-35172018-2653

Agnew, J. (1994). The territorial trap: The geographical assumptions of international relations theory. Review of International Political Economy, 1(1), 53-80. https://doi.org/10.1080/09692299408434268

Agnew, J. (2003). Geopolitics: Re-visioning world politics. Psychology press.

Agnew, J. (2005). Hegemony: The new shape of global power. Temple University Press.

Allen, J. (2003). Lost geographies of power 79. Blackwell Publishing Ltd.

Althusser, L. (1970). Ideología y aparatos ideológicos. Nueva Visión.

Arrighi, G. (1999). El largo siglo XX. Akal Madrid.

Arrighi, G. (2005). Comprender la hegemonía. New Left Review, 32, 20-74. https://newleftreview.es/issues/32/articles/giovanni-arrighi-comprender-la-hegemonia-1.pdf

Arrighi, G. (2007). Adam Smith in Beijing: lineages of the 21st century. Verso.

Bieler, A., \& Morton, A. D. (2004). A critical theory route to hegemony, world order and historical change: Neo-Gramscian perspectives in international relations. Capital \& Class, 28(1), 85-113. https://doi.or $\mathrm{g} / 10.1177 \% 2 \mathrm{~F} 030981680408200106$

Buci-Glucksmann, C. (1978). Gramsci y el Estado: hacia una teoría materialista de la filosofía. Siglo XXI Editores.

Buller, J. (2018). State spatiality in an era of global and regional interdependence: The linkage governance approach. Comparative European Politics, 16(2), 224-248. https://doi.org/10.1057/cep.2015.24

Cardoso, F. H., \& Faletto, E. (1977). Dependência e desenvolvimento na América Latina: Ensaio de interpretação sociologica. Zahar Editores.

Carr, E. H. (2004). La crisis de los veinte años, 1919-1939: una introducción al estudio de las relaciones internacionales 173. Los libros de la Catarata.

Castells, M. (2016). A sociology of power: My intellectual journey. Annual Review of Sociology, 42, 1-19. https://www.annualreviews.org/doi/ abs/10.1146/annurev-soc-081715-074158

Cohen, B. J. (2014). Advanced introduction to international political economy. Edward Elgar Publishing. 
Cox, R. W. (1981). Social forces, states and world orders: beyond international relations theory. Millennium, 10(2), 126-155. https://doi.org/1 $0.1177 / 03058298810100020501$

Cox, R. W. (1983). Gramsci, hegemony and international relations : An essay in method. Millennium, 12(2), 162-175. https://doi.org/10.117 7/03058298830120020701

Cox, R. W. (1987). Production, power, and world order: Social forces in the making of history 1. Columbia University Press.

Cox, R. W. (2013). Fuerzas sociales, estados y órdenes mundiales: Más allá de la teoría de relaciones internacionales. Relaciones Internacionales, (24). https://revistas.uam.es/relacionesinternacionales/article/view/5195

Cox, R. W. (2016). Gramsci, hegemonía y relaciones internacionales: Un ensayo sobre el método. Relaciones Internacionales (31). https: / / revistas. uam.es/relacionesinternacionales/article/view/5301

Deciancio, M. (2018). La economía política internacional en el campo de las Relaciones Internacionales argentinas. Desafíos, 30(2), 15-42. https: / / doi.org/10.12804/revistas.urosario.edu.co/desafios/a.6106

Dos Santos, T. (1986). Imperialismo y dependencia. Ediciones Era.

Fernández, V. R. (2017). La trilogía del erizo-zorro. Redes globales, trayectorias nacionales y dinámicas regionales desde la periferia. Anthropos - Siglo XXI Editores - Ediciones UNL.

Fernández, V. R., \& Moretti, L. (2020). Un nuevo sistema mundo desde el Sur Global: gran convergencia y desplazamiento geografico acelerado. Geopolitica(s). Revista de Estudios Sobre Espacio y Poder, 11(2), 313-345. https://doi.org/10.5209/geop.69203

Gibson Graham, J. K. (1996). The end of capitalism (as we knew it): A feminist critique of political economy. Blackwell Publishers Inc.

Gill, S. (1995). Globalization, market civilization, and disciplinary neoliberalism. Journal of International Studies, 24, 903-910. https://doi.org/10 $.1177 / 03058298950240030801$

Gill, S. (2008). Power and resistance in the new world order. Springer.

Gill, S., \& Law, D. (1989). Global hegemony and the structural power of capital. International Studies Quarterly, 33(4), 475-499. https://doi. org/10.2307/2600523

Glassman, J. (1999). State power beyond the territorial trap': The internationalization of the state. Political Geography, 18(6), 669-696. https:// doi.org/10.1016/S0962-6298(99)00013-X 


\section{4 / Joel Sidler - Víctor Ramiro Fernández}

Gramsci, A. (1981a). Cuadernos de la cárcel. Tomo 3. Ediciones Era.

Gramsci, A. (1981b). Cuadernos de la cárcel. Tomo 5. Ediciones Era.

Harvey, D. (1998). La condición de la posmodernidad. Investigación sobre los orígenes del cambio cultural. Amorrortu Editores.

Harvey, D. (2005). The new imperialism. Oup Oxford.

Herrera Santana, D. (2017). Hegemonía y relaciones internacionales/I Un estado del arte. Revista de Relaciones Internacionales de La UNAM, (127). http://www.revistas.unam.mx/index.php/rri/article/ view/61145/53941

Hoffmann, S. (1966). Obstinate or obsolete? The fate of the nation-state and the case of western Europe. Daedalus, 95(3), 862-915. http:// www.jstor.org/stable/20027004

Ikenberry, J., \& Nexon, D. (2019). Hegemony studies 3.0: The dynamics of hegemonic orders. Security Studies, 28(3), 395-421. https:/ / doi.org /10.1080/09636412.2019.1604981

Jaguaribe, H. (1979). Autonomía periférica y hegemonía céntrica. Estudios Internacionales, 12(46), 91-130. https://doi.org/10.5354/07193769.2011.16458

Krasner, S. D. (2001). Problematic sovereignty: Contested rules and political possibilities. Columbia University Press.

Lamy, S. L. (2001). Contemporary mainstream approaches neo-realism and neoliberalism. En J. Baylis and S. Smith (eds), The Globalisation of World Politics (pp. 205-225). Oxford University Press.

Maquiavelo, N. (1971). Elpríncipe. Ediciones Ibéricas y LCL.

Míguez, P. (2017). Capital global, integración regional y Estados Nacionales en el siglo XXI: más allá de las teorías del imperialismo y de las relaciones internacionales (dossier). Revista Estado y Politicas Públicas, 5(8), 41-57. http://hdl.handle.net/10469/12463

Parnreiter, C. (2018). America first! Donald Trump, the demise of the U.S. hegemony and chaos in the capitalist world-system. Zeitschrift Fur Wirtschaftsgeographie, 62(1), 1-13. https:/ / doi.org/10.1515/zfw-2017-0024

Prebisch, R. (1986). El desarrollo económico de la América Latina y algunos de sus principales problemas. Desarrollo Económico, 26(103), 479-502. https://doi.org/10.2307/3466824

Rey, M. T. (2007). Estado y marxismo: un siglo y medio de debates. Prometeo Libros Editorial. 
Ruggie, J. G. (1982). International regimes, transactions, and change: Embedded liberalism in the postwar economic order. International Organization, 36(2), 379-415.

Sassen, S. (2008). Territory, authority, rights: From medieval to global assemblages. Princeton university press.

Sassen, S. (2009). Bordering capabilities versus borders: Implications for national borders. Michigan Journal of International Law, 30(3), 567-597. https:// repository.law.umich.edu/cgi/viewcontent. cgi? article $=1127 \&$ context $=$ mjil

Sassen, S. (2013). When territory deborders territoriality. Territory, Politics, Governance, 1(1), 21-45. https://doi.org/10.1080/21622671.2013.769895

Sassen, S. (2019). Researching the localizations of the global. En M. Juergensmeyer, S. Sassen, M. B. Steger, \& V. Faessel (Eds.), The Oxford Handbook of Global Studies (pp. 73-92). Oxford University Press.

Tilly, C. (1984). Big structures, large processes, huge comparisons. Russell Sage Foundation.

Tilly, C. (2016). Explaining social processes. Routledge.

Tussie, D., \& Riggirozzi, P. (2015). A global conversation: Rethinking IPE in post-hegemonic scenarios. Contexto Internacional, 37, 1041-1068. https://doi.org/10.1590/S0102-85292015000300009

Wallerstein, I. (1974). The modern world-system: capitalist agriculture and the origins of the European world-economy in the sixteen century. Academic Press.

Wallerstein, I. (1984). The politics of the world-economy: The states, the movements and the civilizations. Cambridge University Press.

Wallerstein, I. (2004). World-systems analysis: An introduction. Duke University Press.

Worth, O. (2011). Recasting Gramsci in international politics. Review of International Studies, 37(1), 373-392. https:/ /www.jstor.org/stable/23024598 